PROCEEDINGS OF THE

AMERICAN MATHEMATICAL SOCIETY

Volume 124, Number 1, January 1996

\title{
A CARTAN THEOREM FOR BANACH ALGEBRAS
}

\author{
THOMAS RANSFORD
}

(Communicated by Theodore Gamelin)

\begin{abstract}
Let $A$ be a semisimple Banach algebra, and let $\Omega_{A}$ be its spectral unit ball. We show that every holomorphic map $G: \Omega_{A} \rightarrow \Omega_{A}$ satisfying $G(0)=0$ and $G^{\prime}(0)=I$ fixes those elements of $\Omega_{A}$ which belong to the centre of $A$, but not necessarily any others. Using this, we deduce that the automorphisms of $\Omega_{A}$ all leave the centre invariant. As a further application, we give a new proof of Nagasawa's generalization of the Banach-Stone theorem.
\end{abstract}

\section{Statement of Results}

Given a (complex, unital) Banach algebra $A$, its spectral unit ball is the set

$$
\Omega_{A}:=\{a \in A: \rho(a)<1\},
$$

where $\rho(a)$ denotes the spectral radius of $a$. It is always an open subset of $A$, though, unlike its norm counterpart, it need be neither convex nor bounded. It arises, for example, in the spectral Nevanlinna-Pick problem (see e.g. [3]), which is of importance in control theory.

A major problem is to identify the holomorphic automorphisms of $\Omega_{A}$, these being the natural analogue of the Möbius automorphisms of the unit disc. In [5], M. C. White and the author studied these automorphisms in the important special case when $A=\mathcal{M}_{n}$, the algebra of $n \times n$ matrices. The main result was that, in contrast with the situation for the unit disc, the automorphism group is not transitive on $\Omega_{\mathcal{M}_{n}}$ if $n \geq 2$. The proof of this was rather intricate and depended on the special properties of matrices. In this paper we shall give a much simpler proof, which at the same time extends to more general Banach algebras. We shall also see that there is a link with a long-standing open problem in Banach-algebra theory.

Our principal tool is the following analogue of Cartan's theorem. We write $I$ for the identity map, and $Z_{A}$ for the centre of a Banach algebra $A$ :

$$
Z_{A}:=\{c \in A: a c=c a \text { for all } a \in A\} .
$$

Theorem 1. Let $A$ be a semisimple Banach algebra.

(a) If $G: \Omega_{A} \rightarrow \Omega_{A}$ is a holomorphic map satisfying $G(0)=0$ and $G^{\prime}(0)=I$, then $G(c)=c$ for all $c \in \Omega_{A} \cap Z_{A}$.

Received by the editors August 24, 1994.

1991 Mathematics Subject Classification. Primary 46Hxx; Secondary 32Hxx.

The author was supported by grants from NSERC and FCAR.

(C)1996 American Mathematical Society 
(b) Given $a \in \Omega_{A} \backslash Z_{A}$, there exists a holomorphic map $G$ : $\Omega_{A} \rightarrow \Omega_{A}$ satisfying $G(0)=0$ and $G^{\prime}(0)=I$ such that $G(a) \neq a$.

The reason why the classical Cartan theorem does not apply to give $G \equiv I$ on the whole of $\Omega_{A}$ is that, as remarked earlier, $\Omega_{A}$ may be unbounded. In fact, using [1, Corollary 5.2.3] one can easily show that $\Omega_{A}$ is bounded if and only if $A$ is isomorphic to a uniform algebra.

Using Theorem 1, we deduce our result on non-transitivity.

Theorem 2. Let $A$ and $B$ be semisimple Banach algebras. If $F: \Omega_{A} \rightarrow \Omega_{B}$ is a biholomorphic map, then $F\left(\Omega_{A} \cap Z_{A}\right)=\Omega_{B} \cap Z_{B}$.

Thus, in particular, if $A=\mathcal{M}_{n}$ and $F$ is an automorphism of $\Omega_{\mathcal{M}_{n}}$, then $F(0)$ is necessarily a multiple of the identity, thereby re-proving the main result of [5].

Returning to Theorem 1, even though we cannot conclude that $G$ fixes points of $\Omega_{A} \backslash Z_{A}$, it is still possible to say something about its action there. This is a consequence of the following version of Schwarz's lemma. We write $\sigma(a)$ for the spectrum of $a$, and $\Gamma_{r}$ for the circle in $\mathbb{C}$ with centre 0 and radius $r$.

Theorem 3. Let $A$ and $B$ be Banach algebras, and let $F: \Omega_{A} \rightarrow \Omega_{B}$ be a holomorphic map such that $F(0)=0$. Then for each $a \in \Omega_{A}$,

$$
\rho(F(a)) \leq \rho(a) \quad \text { and } \quad \rho\left(F^{\prime}(0) a\right) \leq \rho(a),
$$

and

$$
\sigma(F(a)) \cap \Gamma_{\rho(a)}=\sigma\left(F^{\prime}(0) a\right) \cap \Gamma_{\rho(a)} .
$$

Thus, under the hypotheses of Theorem 1, we may conclude that $G(a)$ always has the same peripheral spectrum as $a$, even if $a \in \Omega_{A} \backslash Z_{A}$. One is led naturally to ask if the same might be true of the whole spectrum.

Question 1. Let $A$ be a semisimple Banach algebra and let $G: \Omega_{A} \rightarrow \Omega_{A}$ be a holomorphic map satisfying $G(0)=0$ and $G^{\prime}(0)=I$. Does it follow that $\sigma(G(a))=$ $\sigma(a)$ for all $a \in \Omega_{A}$ ?

If $A$ is the algebra of $n \times n$ matrices, then the answer is yes: this was proved in [5, Theorem 3]. Even if the answer is no in general, perhaps it is still at least true that $\sigma(G(a))$ and $\sigma(a)$ have the same polynomial hull.

Combining the Schwarz lemma with the earlier Cartan theorem leads to our final result. We write $e$ for the identity in a Banach algebra.

Theorem 4. Let $A$ and $B$ be commutative semisimple Banach algebras, and let $F: \Omega_{A} \rightarrow \Omega_{B}$ be a biholomorphic map.

(a) If $F(0)=0$, then $F$ extends to a linear isomorphism $F: A \rightarrow B$.

(b) If also $F(e)=e$, then $F: A \rightarrow B$ is an algebra isomorphism.

Part (b) of this result is originally due to M. Nagasawa [4] (see also [1, §4.1], which explains the connection with the Banach-Stone theorem). The proofs in both these references depend on convexity theory, whereas the complex-variable proof of Theorem 4 given below is quite different.

Once again, one is led to ask if this result has a non-commutative version. The most natural formulation seems to be the following, which has been an open question for about 25 years. 
Question 2. Let $A$ and $B$ be semisimple Banach algebras, and let $F: A \rightarrow B$ be a linear isomorphism such that $F(e)=e$ and $\sigma(F(a))=\sigma(a)$ for all $a \in A$. Does it follow that $F$ is a Jordan isomorphism, i.e. $F\left(a^{2}\right)=F(a)^{2}$ for all $a \in A$ ?

The answer is known to be yes in a number of special cases (see [2], which also contains a brief history of the problem). It is possible that Question 2 might prove more tractable if we had a positive answer to Question 1.

\section{Proofs}

Proof of Theorem 1. (a) Fix $c \in \Omega_{A} \cap Z_{A}$, and consider the function $\lambda \mapsto G(\lambda c)$, which is holomorphic on $\{\lambda \in \mathbb{C}:|\lambda|<1 / \rho(c)\}$. Since $G(0)=0$ and $G^{\prime}(0)=I$, this function has a Taylor expansion about 0 of the form

$$
G(\lambda c)=\lambda c+\sum_{j=2}^{\infty} \lambda^{j} a_{j} \quad(|\lambda|<1 / \rho(c)),
$$

where the $a_{j}$ are elements of $A$. Our aim is to show that $a_{j}=0$ for all $j$. Suppose, for a contradiction, that this is not the case, and let $k$ be the smallest integer such that $a_{k} \neq 0$. Take $q \in A$ with $\rho(q)=0$, and let $n \geq 1$. Then, writing $G^{n}$ for the $n$-fold composition $G \circ \cdots \circ G$, we have

$$
G^{n}\left(\lambda c+\lambda^{k} n q\right)=\lambda c+\lambda^{k} n\left(a_{k}+q\right)+O\left(\lambda^{k+1}\right) \quad \text { as } \lambda \rightarrow 0 .
$$

Now as $c$ and $q$ commute, it follows that $\rho\left(\lambda c+\lambda^{k} n q\right) \leq \rho(\lambda c)+\rho\left(\lambda^{k} n q\right)=|\lambda| \rho(c)$ (see e.g. [1, Corollary 3.2.10]), and so we can define a holomorphic function $g:\{0<|\lambda|<1 / \rho(c)\} \rightarrow A$ by

$$
g(\lambda)=\frac{G^{n}\left(\lambda c+\lambda^{k} n q\right)-\lambda c}{n \lambda^{k}} \quad(0<|\lambda|<1 / \rho(c)) .
$$

From (2) we see that the isolated singularity at $\lambda=0$ can be removed by setting $g(0)=a_{k}+q$. By Vesentini's theorem (see e.g. [1, Theorem 3.4.7]), the composition $\rho \circ g$ is a subharmonic function on $\{|\lambda|<1 / \rho(c)\}$, and so by the maximum principle

$$
\rho(g(0)) \leq \max _{|\lambda|=1} \rho(g(\lambda)) .
$$

Making use of [1, Corollary 3.2.10] again to estimate the right-hand side, it follows that

$$
\rho\left(a_{k}+q\right) \leq 2 / n \text {. }
$$

As this is true for each $n$, we can let $n \rightarrow \infty$ deduce that $\rho\left(a_{k}+q\right)=0$. And as this holds for each $q \in A$ with $\rho(q)=0$, Zemánek's characterization of the radical [1, Theorem 5.3.1] implies that $a_{k}$ belongs to the radical of $A$, which is zero since $A$ is semisimple. Thus $a_{k}=0$, and we have arrived at a contradiction. We conclude that indeed $a_{j}=0$ for all $j \geq 2$, and hence from (1) that $G(c)=c$.

(b) Let $a \in \Omega_{A} \backslash Z_{A}$. Then there exists $u \in A$ such that $a u \neq u a$. We can suppose that $\|u\|<1$. Then $v:=\log (e-u)$ satisfies $e^{-v} a e^{v} \neq a$. Choose a continuous linear functional $\phi$ on $A$ such that $\phi(a)=1$, and define $G: \Omega_{A} \rightarrow \Omega_{A}$ by

$$
G(x)=e^{-\phi(x) v} x e^{\phi(x) v} \quad\left(x \in \Omega_{A}\right) .
$$

Then $G$ is holomorphic, $G(0)=0$ and $G^{\prime}(0)=I$, but $G(a)=e^{-v} a e^{v} \neq a$. 
Proof of Theorem 2. Fix $c \in \Omega_{A} \cap Z_{A}$, and suppose also, for the moment, that $c \neq 0$. Then $F(c) \neq F(0)$, so there exists a continuous linear functional $\psi$ on $B$ such that $\psi(F(c)-F(0))=1$. Take $b \in B$, and define $C: \Omega_{B} \rightarrow \Omega_{B}$ by

$$
C(y)=e^{-\psi(y-F(0))^{2} b} y e^{\psi(y-F(0))^{2} b} \quad\left(y \in \Omega_{B}\right) .
$$

Then $C$ is holomorphic, $C(F(0))=F(0)$ and $C^{\prime}(F(0))=I$. Hence if we set $G=F^{-1} \circ C \circ F$, then $G$ is a holomorphic self-map of $\Omega_{A}$ satisfying $G(0)=0$ and $G^{\prime}(0)=I$. By Theorem 1, it follows that $G(c)=c$, so that $C(F(c))=F(c)$ or, in other words $e^{-b} F(c) e^{b}=F(c)$. This holds for every $b \in B$, so as in the proof of part (b) of Theorem 1, we deduce that $F(c) \in Z_{B}$. This has been proved under the assumption that $c \neq 0$, but by continuity it remains true if $c=0$. Thus $F\left(\Omega_{A} \cap Z_{A}\right) \subset \Omega_{B} \cap Z_{B}$, and the reverse inclusion follows by applying the same argument to $F^{-1}$.

Proof of Theorem 3. Fix $a \in \Omega_{A}$, and define $f:\{0<|\lambda|<1 / \rho(a)\} \rightarrow B$ by

$$
f(\lambda)=F(\lambda a) / \lambda \quad(0<|\lambda|<1 / \rho(a)) .
$$

Then $f$ is holomorphic, and since $F(0)=0$ we can remove the isolated singularity at $\lambda=0$ by setting $f(0)=F^{\prime}(0) a$. Now using Vesentini's theorem once again, the function $\rho \circ f$ is subharmonic on $\{|\lambda|<1 / \rho(a)\}$, and so for each $r<1 / \rho(a)$ it follows from the maximum principle that

$$
\rho(f(\lambda)) \leq \max _{|\mu|=r} \rho(f(\mu)) \leq 1 / r \quad(|\lambda| \leq r) .
$$

Letting $r \rightarrow 1 / \rho(a)$, we obtain

$$
\rho(f(\lambda)) \leq \rho(a) \quad(|\lambda|<1 / \rho(a)) .
$$

In particular, taking $\lambda=1$ and $\lambda=0$, we deduce that $\rho(F(a)) \leq \rho(a)$ and $\rho\left(F^{\prime}(0) a\right) \leq \rho(a)$, which proves the two inequalities in the theorem. (This half of the proof was essentially the same as in [5, Theorem 2].)

Now fix $\zeta \in \mathbb{C}$ with $|\zeta|=\rho(a)$. Then we have $\rho(f(\lambda)+\zeta e) \leq 2 \rho(a)$ for all $\lambda$, with equality if and only if $\zeta \in \sigma(f(\lambda))$. However, by Vesentini's theorem once more, the function $\lambda \mapsto \rho(f(\lambda)+\zeta e)$ is subharmonic, so if it attains a maximum, then it must be constant. Therefore if $\zeta \in \sigma(f(\lambda))$ for one value of $\lambda$, then the same holds for all $\lambda$. The conclusion is that the set $\sigma(f(\lambda)) \cap \Gamma_{\rho(a)}$ is independent of $\lambda$. In particular, taking $\lambda=1$ and $\lambda=0$ we obtain $\sigma(F(a)) \cap \Gamma_{\rho(a)}=\sigma\left(F^{\prime}(0) a\right) \cap \Gamma_{\rho(a)}$, which proves the final statement of the theorem.

Proof of Theorem 4. (a) By the second inequality of Theorem 3, applied both to $F$ and $F^{-1}$, we see that $\left.F^{\prime}(0)\right|_{\Omega_{A}}$ is a biholomorphic map of $\Omega_{A}$ onto $\Omega_{B}$. Hence if we set $G=\left.F^{-1} \circ F^{\prime}(0)\right|_{\Omega_{A}}$, then $G$ is a holomorphic self-map of $\Omega_{A}$ satisfying $G(0)=0$ and $G^{\prime}(0)=I$. From Theorem 1, together with the fact that $A$ is commutative, it follows that $G(a)=a$ for all $a \in \Omega_{A}$, and so $F \equiv F^{\prime}(0)$ on $\Omega_{A}$. Thus $F^{\prime}(0)$ is a linear isomorphism of $A$ onto $B$ which extends $F$.

(b) Assume now that $F$ has been so extended, and that $F(e)=e$. For $t \in(-1,1)$, define $M_{t}: \Omega_{A} \rightarrow \Omega_{A}$ and $N_{t}: \Omega_{B} \rightarrow \Omega_{B}$ by

$$
\begin{aligned}
M_{t}(x) & =(x-t e)(e-t x)^{-1} & & \left(x \in \Omega_{A}\right), \\
N_{t}(y) & =(y-t e)(e-t y)^{-1} & & \left(y \in \Omega_{B}\right) .
\end{aligned}
$$

Then $M_{t}$ is biholomorphic, with $M_{t}(0)=-t e$ and $M_{t}^{\prime}(0)=\left(1-t^{2}\right) I$, and similarly for $N_{t}$. Also, since $F$ is linear and maps $e$ to $e$, we have $F(-t e)=-t e$. Hence if we 
define $G=F^{-1} \circ N_{t}^{-1} \circ F \circ M_{t}$, then $G$ is a holomorphic self-map of $\Omega_{A}$ satisfying $G(0)=0$ and $G^{\prime}(0)=I$. Applying Theorem 1 once more, we deduce that $G(a)=a$ for all $a \in \Omega_{A}$, or in other words that $F\left(M_{t}(a)\right)=N_{t}(F(a))$ for all $a \in \Omega_{A}$. Now fix $a \in \Omega_{A}$, and expand both sides of this last equation in powers of $t$. This gives

$$
F(a)+t F\left(a^{2}-e\right)+O\left(t^{2}\right)=F(a)+t\left(F(a)^{2}-e\right)+O\left(t^{2}\right) \quad \text { as } t \rightarrow 0,
$$

and equating the coefficients of $t$ we obtain $F\left(a^{2}\right)=F(a)^{2}$. Applying this with $a=\frac{1}{2}\left(a_{1} \pm a_{2}\right)$, we deduce that $F\left(a_{1} a_{2}\right)=F\left(a_{1}\right) F\left(a_{2}\right)$ for all $a_{1}, a_{2} \in \Omega_{A}$, and hence, by homogeneity, for all $a_{1}, a_{2} \in A$. This completes the proof.

\section{REFERENCES}

[1] B. Aupetit, A primer on spectral theory, Springer-Verlag, New York, 1991. MR 92c:46001

[2] B. Aupetit and H. du T. Mouton, Spectrum-preserving linear mappings in Banach algebras, Studia Math. 109 (1994), 91-100. MR 95c:46070

[3] H. Bercovici, C. Foias, and A. Tannenbaum, A spectral commutant lifting theorem, Trans. Amer. Math. Soc. 325 (1991), 741-763. MR 91j:47006

[4] M. Nagasawa, Isomorphisms between commutative Banach algebras with an application to rings of analytic functions, Kodai Math. Sem. Rep. 11 (1959), 182-188. MR 22:12379

[5] T. J. Ransford and M. C. White, Holomorphic self-maps of the spectral unit ball, Bull. London Math. Soc. 23 (1991), 256-262. MR 92g:32049

Département de Mathématiques et de Statistique, Université Laval, Québec (QC), CANADA G1K 7P4

E-mail address: ransford@mat.ulaval.ca 